\title{
Editorial
}

Int Neurourol J 2013;17:153-154

http://dx.doi.org/10.5213/inj.2013.17.4.153

pISSN 2093-4777 · eISSN 2093-6931

\section{More Than the Significance of Conventional Urinalysis}

\author{
Khae Hawn Kim \\ Associate Editor \\ Department of Urology, Gachon University Gil Medical Center, Incheon, Korea \\ E-mail: kimcho99@gilhospital.com
}

It is nowadays well established that a urinalysis is one of the most essential and useful diagnostic modalities in the field of urology. On many occasions, however, its key elements are neglected and omitted and the inaccurate results are produced and then misinterpreted accordingly. This occurs when the investigators collect inappropriate samples, perform the test incompletely during short periods of time, possess a lower level of technical expertise, and neglect the significance of results. Currently, there is a widespread recognition that a microscopic urinalysis would be unnecessary so long as if there are normal results on dip-strip test that is routinely performed as a macroscopic one. But if there are any chances that specific markers, such as protein, hemoglobin, leukocyte esterase or nitrate, are detected on dip-strip test, it would be mandatory to perform a complete urinalysis including a microscopic one. A macroscopic urinalysis is routinely performed to examine the color and appearance, specific gravity, chemical composition test for $\mathrm{pH}$, protein, glucose, hemoglobin, bacteria (nitrate), and leukocyte esterase. On the other hand, a microscopic urinalysis is performed to detect bacteria, leukocyte, erythrocytes, epithelial cells, and casts. Moreover, it may also be used to make a diagnosis of colon cancer, diverticulitis, and regional ileitis in patients with fistula beyond urothelial cancer, prostate cancer, hormone, and stone constituents on urinalysis. But I do assume that various types of qualitative and quantitative test regimens might be clinically useful only in a limited scope because of their sensitivity, specificity and high cost. In an actual clinical setting, bladder tumor antigen, nuclear matrix protein 22, immunCyt, and uroVysion fluorescence in situ hybridization are also currently available as adjunct to cytology in making a diagnosis of urothelial cancer. In addition, prostate cancer antigen 3, also known as differential display code 3 , has been of increasing interest as a novel biomarker for prostate cancer, and ongoing studies are conducted to examine its clinical significance. Current efforts are made to identify and to examine the clinical application of novel urine markers in making a diagnosis of lower urinary tract dysfunction (LUTD) and other related diseases. But this is greatly dependent on the discovery of novel target markers rather than the simple application of new technology. It would be both complex and time-consuming to screen and to establish a diagnosis of many conditions associated with LUTD. This is because underlying diseases pose challenging problems for clinicians. It is therefore imperative that efficient biomarkers be discovered and then applied to an actual clinical setting.

In the review of this issue [1], as a follow-up review of the article "Recent progress in lab-on-a-chip technology and Its potential application to clinical diagnoses" published in the first issue of this year [2], the authors describe that a quantitative, microfluidic paper-based bio-analysis is a novel technology and it is also one of the most relevant applications that have overcome the disadvantages of conventional macroscopic urinalysis using a dip strip. This puts an emphasis on the emergence of new technology that has undergone great advancements. Henceforth, we'll make every effort to identify neuro-urologic target markers and to assess their feasibility from medical, scientific and economic perspectives rather than to develop new technologies. 
IN $\mathrm{Kim}$ - More Than the Significance of Conventional Urinalysis

\section{REFERENCES}

1. Jeong SG, Kim J, Nam JO, Song YS, Lee CS. Paper-based analytical device for quantitative urinalysis. Int Neurourol J 2013;17:155-61.

2. Lee NY. Recent progress in lab-on-a-chip technology and its potential application to clinical diagnoses. Int Neurourol J 2013;17:2-10. 